\title{
Integration of blue-green electroluminescence structure in mid-infrared hollow optical fiber for targeting invisible $\mathrm{CO}_{2}$ laser beam
}

\section{Yang Shen}

East China Normal University

\section{Guishun Li}

East China Normal University

\section{Guoxing Xie}

East China Normal University

Changkun She

East China Normal University

Chengbin Jing ( $\nabla$ cbjing@ee.ecnu.edu.cn )

East China Normal University https://orcid.org/0000-0002-6710-2955

Junhao Chu

Fudan University

\section{Research Article}

Keywords: mid-infrared hollow optical fiber, $\mathrm{CO} 2$ laser, visible beam, electroluminescence, ZnS: Cu

Posted Date: February 11th, 2022

DOI: https://doi.org/10.21203/rs.3.rs-1079115/v1

License: (c) (i) This work is licensed under a Creative Commons Attribution 4.0 International License.

Read Full License 


\section{Abstract}

Mid-infrared (IR) $\mathrm{CO}_{2}$ lasers have important applications in laser medicine and material processing. However, the $\mathrm{CO}_{2}$ laser beam is invisible to human eyes, it is not easy to accurately indicate the beam position in practical applications. In this work, we designed a PEDOT (Poly (3, 4-ethylenedioxythiophene)) /ZnS: $\mathrm{Cu} / \mathrm{BaTiO}_{3} / \mathrm{Ag}$ electroluminescence (EL) structure and integrated it at the output end of the mid-IR hollow optical fiber. The blue-green light can be output along with the $\mathrm{CO}_{2}$ laser beam in this EL structure integrated fiber, thus achieving the optical path indication and illumination of invisible laser beam. This work provides a possible guide for aiming the $\mathrm{CO}_{2}$ laser beam in the min-IR optical fiber.

\section{Introduction}

Mid-infrared (IR) $\mathrm{CO}_{2}$ lasers are widely used in laser surgery, rehabilitation treatment, and material processing due to its high gain coefficient, energy conversion efficiency and output power (Hongo et al. 1991; Merberg, 2010; Wang et al. 2017; Zhang et al. 2017). It is mainly transmitted through the various optical fibers. Among them, the hollow optical fiber has attracted much attention because of its excellent performance (Jaworski et al. 2020). Compared with the solid-core fiber, the hollow optical fiber has no end reflection, high laser threshold, low transmission loss, low divergence and good flexibility (Harrington, 2004). Its unique air-core fiber can transmit high-intensity lasers, with a low-cost and simple preparation method(Shi et al. 1999).

According to the transmission principle (Harrington, 2000), the hollow optical fiber can be divided into attenuated total reflection (ATR) fiber and leaky-type fiber. For the former, the laser propagates through total reflection $\left(n_{r}<1\right)$ between the reflective layer and the air interface in ATR fibers. The leaky-type fiber is mainly composed of air, the dielectric layer with suitable thickness, and the metal high reflective layer (Yu et al. 2020). The laser beam is transmitted through continuous reflection at the metal-dielectric layer and the air-dielectric layer $\left(n_{r}>1\right)$.

In several applications such as material processing and laser medicine, the mid-IR laser beam is invisible to human eyes (Tichindelean \& Teodor, 2016), so it is not easy to target the laser-beam position in the operation process. Moreover, the typical optical fiber used to transmit the mid-IR light has a low loss for $\mathrm{CO}_{2}$ laser but a high loss for the visible light band. The visible beam can hardly be transmitted to the target position when the $\mathrm{CO}_{2}$ laser is delivered. Therefore, it is urgent to explore an approach to aim the laser beam position. In the early years, many researchers solved this problem by adding a visible light path. (Van Den et al. 1992) However, the red aiming beam used in this traditional auxiliary indicating light path method has low integration, and is difficult to be distinguished by human eyes in laser medical applications (Graham \& Bartlett, 1939; Sheinis et al. 1994). More importantly, when the direction of the $\mathrm{CO}_{2}$ laser-beam needs to be changed, the method that realizes the visible collimating transmission-light path by the optical-lens group will not be applicable. 
In 1996, James A. Harrington's group (Rabii \& Harrington, 1996) proposed a dual-core fiber structure. The optical fiber consists of air core, Ag/Agl hollow glass-fiber layer, quartz-glass-tube layer, and the outermost fluorine-containing low refractive-index layer. The quartz glass tube layer and the outermost fluorine-containing low refractive-index layer can establish an ATR waveguide structure for visible-light transmission. The air core and the Ag/Agl layer on the inner surface build a leaky waveguide structure for transmitting the $\mathrm{CO}_{2}$ laser. This dual-core optical fiber has high structural integration. However, it needs to deposit a fluorine-containing low refractive-index layer on the inner surface of the quartz glass tube by the chemical vapor deposition method and fabricate capillary hollow glass fibers using melt-drawing process. Thus, the production cost is high, leading to the restriction of commercial applications.

In 2016, our research group and Harrington's team (Jing et al. 2016) improved the integration method for manufacturing the dual-core fiber. This optical fiber was manufactured entirely based on the standard quartz glass tubing materials available in the market and the standard manufacturing procedure of $\mathrm{Ag} / \mathrm{Agl}$ hollow glass fibers, rather than the fluorine-doped quartz glass capillary. It significantly reduced the manufacturing cost of the dual-core fiber. Nevertheless, it is still challenging to couple the $\mathrm{CO}_{2}$ laser beam and the visible laser beam simultaneously into the input end of dual-core fiber in practical applications.

Electroluminescence (EL) technology (Picciolo et al. 2001) has a wide spectral range of the visible light region, high luminous intensity, self-luminescence, and fast response (tens of nanoseconds) (Allen, 1994). This work aims to fabricate an EL structure-integrated mid-IR hollow optical fiber, which is capable of emitting high-reliability visible light and transmits $\mathrm{CO}_{2}$ laser by hollow optical fiber. We also investigated the fabrication process and the visible light targeting results of the fiber for the invisible $\mathrm{CO}_{2}$ laser beam. The research work shows that the EL integrated Ag/Agl hollow optical fiber is able to visualize the invisible $\mathrm{CO}_{2}$ laser output beam which may be potential in laser surgery and materials processing applications.

\section{Experiments}

Figure 1 shows the structure of the EL integrated Ag/Agl hollow optical fiber. The Ag/Agl air-core waveguide structure can serve for transmitting $\mathrm{CO}_{2}$ laser. PEDOT (Poly (3, 4-ethylenedioxythiophene)) /ZnS: $\mathrm{Cu} / \mathrm{BaTiO}_{3} / \mathrm{Ag}$ EL structure is integrated at the output end of the hollow optical fiber. Visible light is generated and enters the air-core and outputs along with the $\mathrm{CO}_{2}$ laser beam. EL blue-green light is selected since it is sensitive to human eyes and easy to distinguish from the background color of blood red human tissue.

The quartz glass tubes were purchased from Ruifeng optical fiber Company (inner diameter: $530 \mu \mathrm{m}$, length: $1 \mathrm{~m}$ ). The ZnS: Cu luminescent powders and epoxy resin were bought from Shanghai Kerun optoelectronics Co., Ltd. 
$\mathrm{Ag} / \mathrm{Agl}$ hollow optical fibers were prepared by using a dynamic liquid phase deposition (DLPD) method (Harrington, 2004; Jing et al. 2016; Navarro-Cía et al. 2015) (See Fig. 2a). First, stannous chloride solution was pumped into a quartz glass tube by a peristaltic pump to pretreat the inner surface of the substrate tube. Then, the silver ammonia solution and reduced dextran solution were pumped into the glass tube to deposit a silver film on the inner tube wall. The thickness of the silver film is controlled by adjusting the reaction time. Finally, the iodine cyclohexane solution was pumped into the silver-coated glass tube and the top layer of the silver film was converted into a silver iodide layer. The iodization process lasted for 6 min to obtain an $\mathrm{Agl}$ layer with an optimized thickness for $10.6 \mu \mathrm{m} \mathrm{CO}_{2}$ laser.

1-cm long polyimide coating and the Ag/Agl film on the inner wall at the output end of the as-deposited $\mathrm{Ag} / \mathrm{Agl}$ hollow optical fiber were removed by burning method. Then, the purchased PEDOT conductive liquid was coated on the $1.2 \mathrm{~cm}$ part of the outer wall at the optical-fiber end by dip-coating (See Fig. 2b). The extended $0.2 \mathrm{~cm}$ PEDOT conductive layer was exposed without contacting the subsequent EL structure layer, convenient to extract the copper wire for the front electrode. Finally, we put it into a $40^{\circ} \mathrm{C}$ oven to dry (about $20 \mathrm{~min}$ ).

$0.4 \mathrm{~g}$ epoxy resin adhesive, $0.15 \mathrm{~g}$ dimethylformamide, and $0.4 \mathrm{~g} \mathrm{ZnS:} \mathrm{Cu} \mathrm{luminescent} \mathrm{powder} \mathrm{were}$ mixed by stirring. The prepared luminescent powder mixture was coated on the transparent part of the hollow optical-fiber end by dip-coating method. The luminescent layer was coated three times by the same method with the $40 \mu \mathrm{m}$ thickness of the three-layer fluorescent layer. It is noted that the prepared luminescent layer had no leakage point so that the front and back electrodes were not easy to break down and cause a short circuit. The main component of dielectric powder is barium titanate with a high dielectric constant, whose dilution ratio and coating method are the same as those of the fluorescent layer. The coating process was repeated four times, and the thickness of the dielectric layer was $60 \mu \mathrm{m}$. After each dip-coating, it was dried in an oven at $60^{\circ} \mathrm{C}$ (about $30 \mathrm{~min}$ ). The conductive silver paste was dipped with a brush and coated on the dielectric layer's outer wall as a back electrode, then dried in an oven. Finally, the PEDOT-based front electrode and the conductive silver paste-based back electrode were extracted with 20- $\mu \mathrm{m}$-diameter copper wire, respectively. The whole optical fiber was wrapped with a transparent insulating heat-shrinkable tube and heated at $60^{\circ} \mathrm{C}$ to form a protective polymer insulatinglayer to ensure the device's insulation and chemical stability.

The surface morphology and crystal structure of ZnS: Cu were determined by X-ray diffraction (XRD: Rigaku, Ultima IV) and scanning electron microscope (SEM: Zeiss, Gemini 450). The visible EL spectrum was measured by the fluorescence spectrophotometer (LengGuang, F97PRO). The $\mathrm{CO}_{2}$ laser beam was generated by the coherent $\mathrm{C} 30$ a laser device and coupled into the waveguide sample after being focused by a ZnSe lens with a focal length of $10 \mathrm{~cm}$. The output power was measured by the laser power detector (LP-3C). The transmission loss of the waveguide samples was calculated by the truncation method. The output laser beam profile and full divergence angle (FDA) of the waveguide samples were measured by the beam quality analyzer (LaserDec CL200). The loss spectra of waveguide samples was measured by a Fourier Transform IR spectrometer (FT-IR: BRUKER, VERTEX 80/80v). 


\section{Results And Discussion}

The ZnS: Cu powder was used for the electroluminescent structure. Fig. 3a shows that the particle size is about 17 55 $\mu \mathrm{m}$. According to energy dispersive spectrometer (EDS), the elemental composition of the electroluminescent structure phosphor is $\mathrm{Zn}, \mathrm{S}$, and $\mathrm{Cu}$.

Figure $3 \mathrm{~b}$ shows the EL cross-sectional SEM morphology at the end of the EL structure-integrated mid-IR hollow optical fiber. The heat-shrinkable tube layer for protection has been removed to capture the crosssection of the optical fiber for the SEM investigation. It can be found that the device has a sandwich structure, and the PEDOT conductive film, the luminescent layer, the dielectric layer, and the silver back electrode layer are on the outer surface of the hollow fiber glass tube in turn. Since the adhesive used in the luminescent layer and the dielectric layer is the same epoxy resin adhesive, the hierarchical structure is closely bonded with blurred boundary.

Figure 4 shows XRD pattern of the ZnS: Cu powder. The XRD patterns of the samples were compared with the standard pattern of ZnS. The diffraction peaks of ZnS: Cu powder were partially consistent with the standard patterns of ZnS-Sphalerite and ZnS-Wurtzite-2H (Yang et al. 2010). The luminescent layer power used in the work includes cubic phase and hexagonal phase.

We measured the visible light emission intensity of waveguide sample by changing the driving voltage with the transformer and illuminometer (See Fig. 5a). It indicates that the light intensity is positively correlated to the voltage. When the voltage exceeds $110 \mathrm{~V}$, the luminescence of the prepared EL structure will be unstable, and it can be as a prone to high-voltage breakdown. When the voltage is $110 \mathrm{~V}$, the luminescence of the EL structure can maintain $168.6 \mathrm{~cd} / \mathrm{m}^{2}$ without breakdown. Although the required frequency and voltage are high, the load current is small (about 0.016-0.3 mA). Therefore, the power consumption is low. In addition, during the preparation of the EL structure, the heat shrinkable tube was used in this work to encapsulate it without the risk of leakage. Considering the luminescence and stability of the EL structure, $110 \mathrm{~V}$ AC voltage is selected to supply power for EL structure.

In the AC state, the T2 or E level of shallow donor electrons and copper ions in ZnS molecules will transition (Liang et al. 2017), thus emitting visible beam. At 110 V AC voltage, the EL spectrum analysis results are shown in Fig. 5b. It can be seen that the luminescent wavelength range of the EL structure is $400-600 \mathrm{~nm}$, and the emission peak is located at $457 \mathrm{~nm}$, belonging to the blue-green light.

Figure 6 shows the loss spectra of the mid-IR fiber with and without the EL structure (the length: $30 \mathrm{~cm}$ ). As expected, both of them possess low-loss windows at $10.6 \mu \mathrm{m}$, which are suitable for the transmitting $\mathrm{CO}_{2}$ laser (Rabii et al. 1999). The transmission loss of the mid-IR fiber with EL structure is slightly higher than that of the mid-IR fiber without EL structure, and the shape and position of the loss spectrum of the two optical fibers are the same. It implies that the EL structure only increases the transmission loss of the hollow optical fiber, without affecting the position of the low-loss window of the optical fiber. 
Figure 7 shows the energy distribution (2D and 3D) of the $\mathrm{CO}_{2}$ laser beam of samples. The two energy distribution profiles are almost circular, indicating that the output $\mathrm{CO}_{2}$ laser beams' energy distribution is close to Gaussian distribution. For the 530- $\mu$ m-inner-diameter EL structure-integrated mid-IR hollow optical fiber, the laser beam is still transmitted in the fundamental mode (HE11 mode). The transmission loss value tends to be high, as the inner diameter of the hollow fiber decreases. Some higher-order modes with high loss gradually decay during the beam's transmission, even disappear before reaching the output end of the fiber, and the lower loss of the fundamental mode has a large probability of output through the whole fiber core. This reflects the confinement effect of small-core hollow fiber on high-order modes, which is consistent with the results in Figure 7. It also implies that the integrated EL structure at the end of mid-IR hollow fiber has no significant impact on the transmission mode of hollow fiber.

Then, we compared the straight transmission loss of $\mathrm{CO}_{2}$ laser transmission in waveguide samples with and without the EL structure, and the measured transmission losses were 1.27 and $1.20 \mathrm{~dB} / \mathrm{m}$, respectively. The capillary tube with a 530- $\mu \mathrm{m}$ inner diameter used in this experiment was irregular in diameter. Besides, the vibration caused by truncation in the test of straight transmission loss made the optical fiber produce small displacement. These may have adverse effects on the transmission characteristics of the optical fiber. However, since the fiber's diameter is only $530 \mu \mathrm{m}$, the fiber's output can reach $90 \mathrm{~mW}$, with the high energy density of the output $\mathrm{CO}_{2}$ spot. The energy density can be enough to meet several general laser medical treatments, such as skin surface treatment and laser rehabilitation physiotherapy. Moreover, the optical fiber with a laser transmission loss of less than $2 \mathrm{~dB} / \mathrm{m}$ has practical value (Sun et al. 2018). Therefore, the as-fabricated EL structure-integrated mid-IR hollow optical fiber has the practical potential to transmit the $\mathrm{CO}_{2}$ laser beam.

In practical applications, the FDA is a crucial parameter for focusing and collimating the output beam, so it has an essential impact on the laser beam propagation ability and sufficient working distance (Bryce et al. 2011; Hidaka, 1982). By measuring the laser beam's spot sizes along the optical propagation direction, for the mid-IR fiber with/without the EL structure, the FDA's values are 26 and 16 mrad, respectively. When the EL structure is integrated on the mid-IR hollow fiber, the Ag/Agl waveguide reflection layer within $1 \mathrm{~cm}$ at the end of fiber is removed so that the visible light emitted by the EL structure can penetrate into the quartz glass capillary of the fiber core and output from the end of the fiber. Therefore, when the $\mathrm{CO}_{2}$ laser beam is transmitted through the core to the tail end, it will lose the constraint of the $\mathrm{Ag} / \mathrm{Agl}$ waveguide reflector, and tend to diverge to a certain extent, resulting in an increase of the FDA value of output beam. However, considering that the divergence angle is $26 \mathrm{mrad}$, the beam can still be regarded as approximately linear propagation within a limited distance, which may have a negligible effect on the energy density of the spot in practical applications such as laser surgery.

In material processing or laser surgery, it is likely to involve $\mathrm{CO}_{2}$ laser-beam transmission under bending conditions. We measured the bending loss of $\mathrm{CO}_{2}$ laser conducted by EL structure-integrated mid-IR hollow optical fiber with an inner diameter of $530 \mu \mathrm{m}$, by keeping the curvature radius of $10 \mathrm{~cm}$ and input power of $108 \mathrm{~mW}$, and changing the bending angle. 
Figure 8 shows the test results. When the bending angle increases from 0 to $240^{\circ}$, the optical fiber's corresponding output power decreases from 90 to $50 \mathrm{~mW}$, and the transmission loss increases from 1.27 to $5.33 \mathrm{~dB} / \mathrm{m}$. The transmission mode of optical fiber is very sensitive to the bending degree of optical fiber. With an increase of bending degree, the trend of exciting high-order modes increases. High-order modes generally have high transmission loss, so the bending loss of optical fiber will increase with the increase of bending angle.(Matsuura et al. 1994) Nevertheless, in the above bending test process, the power of output laser beam in optical fiber with an internal diameter of $0.53 \mathrm{~mm}$ can reach $50 \sim 90 \mathrm{~mW}$. Considering that its internal diameter is only $0.53 \mathrm{~mm}$ with high energy density in the output $\mathrm{CO}_{2}$ laser spot, it still has practical potential for the general laser medical surgery or laser irradiation rehabilitation therapy. When the bending radius is controlled at $10 \mathrm{~cm}$ and the bending angle is controlled at $0 \sim 50^{\circ}$, the transmission loss is less than $2 \mathrm{~dB} / \mathrm{m}$. Therefore, it suggests the application potential of transmitting $\mathrm{CO}_{2}$ laser under bending conditions.

Figure 9a shows the encapsulated optical fiber. The EL structure-integrated mid-IR hollow optical fiber can emit visible guiding light continuously under 110V AC voltage. It should be noted that the circuit is safe and stable under the encapsulation state, which retains the optical fiber's original flexibility and has high integration. When the optical fiber is transmitted in any direction, the position of the output $\mathrm{CO}_{2}$ laser spot can still be indicated.

Through the experiment of surgical burning with $\mathrm{CO}_{2}$ lasers, we measured the visible light indication effect of IR hollow fiber with inner diameter of $0.53 \mathrm{~mm}$ for $\mathrm{CO}_{2}$ laser transmission, as shown in Fig. $9 \mathrm{~b}, \mathrm{c}$. Since the EL structure is only integrated within the length of $1 \mathrm{~cm}$ from the tail end of the mid-IR optical fiber, the bending state of the optical fiber has little effect on the output of visible light. Red paperboard is selected to simulate the red blood environment during $\mathrm{CO}_{2}$ laser surgery. Two copper wires are externally connected with $\mathrm{AC}$ power supply to excite the $\mathrm{EL}$ structure to produce indicator light. The $\mathrm{CO}_{2}$ laser is coupled into the interior of the EL structure-integrated mid-IR hollow optical fiber for beam transmission. It is found that the EL structure can stably emit the blue-green visible beam while transmitting $\mathrm{CO}_{2}$ lasers. Comparing Fig. $9 \mathrm{~b}$ with Fig. $9 \mathrm{c}$, when the optical fiber sample transmits $\mathrm{CO}_{2}$ lasers, the burning spot of the laser basically coincides with the indicating light spot, located at the center of the indicating light spot. The result shows that the $\mathrm{CO}_{2}$ laser and blue-green visible light can be output to the same position within a certain distance, so that the burning position of the $\mathrm{CO}_{2}$ laser can be determined to prevent accidental injury to other areas.

\section{Conclusion}

By integrating the EL structure into Ag/Agl hollow optical fiber, we successfully fabricated the EL structure-integrated mid-IR hollow optical fiber. The fiber could accurately indicate the output position of $\mathrm{CO}_{2}$ laser with the visible light intensity of $168.6 \mathrm{~cd} / \mathrm{m}^{2}$. The results indicate that the optical fiber could continuously carry out visible light indication with $\mathrm{CO}_{2}$ laser output. Besides, it can stably emit blue-green 
light sensitive to human eyes and possess a good visible light indication effect. It is expected to be applied in $\mathrm{CO}_{2}$ laser medical treatment and material processing.

The results showed that the output of $530 \mu \mathrm{m}$ inner diameter EL structure-integrated mid-IR hollow optical fiber presented the Gaussian distribution. The straight transmission loss of $\mathrm{CO}_{2}$ transmission of the waveguide sample was as low as $1.27 \mathrm{~dB} / \mathrm{m}$, and the output beam FDA was 26 mrad. The output $\mathrm{CO}_{2}$ laser had high quality, and the bending transmission ability could meet practical application requirements.

\section{Declarations}

\section{Acknowledgements}

This work was supported by the National Natural Science Foundation of China (NSFC) (grant numbers $61775060,61275100)$

\section{Declaration of conflicting interests}

The authors declared no potential conflicts of interest with respect to the research, authorship and/or publication of this article.

\section{References}

1. Hongo, A., Morosawa, K., Shiota, T., Suzuki, K., Iwasaki, S., Miyagi, M.: Transmission of 1 kw-class $\mathrm{CO}_{2}$-laser light through circular hollow waveguides for material processing. Appl. Phys. Lett. 58(15), 1582-1584 (1991). doi:10.1063/1.105131

2. Merberg, G.N.: Current status of infrared fiber optics for medical laser power delivery. Laser. Surg. Med. 13(5), 572-576 (1993). doi:10.1002/Ism.1900130513

3. Wang, X., Wang, L., Fu, X.H., Jing, C.B., Yue, F.Y., Yang, P.X., Chu, J.H.: Thermal behaviors of stainless steel tube based $\mathrm{GeO}_{2}$ ATR hollow fibers for transmitting $\mathrm{CO}_{2}$ laser radiations. Opt. Laser Technol. 95, 42-45 (2017). doi:10.1016/j.optlastec.2017.03.045

4. Zhang, J., Zhang, W., Pan, X.M., Huang, S.S., Liu, W.W.: Research on the welding process of aluminum alloy based on high power fiber laser. Proceedings of SPIE Pacific rim laser damage 2017-optical materials for high-power lasers. Pacific Rim Laser Damage Symposium on Optical Materials for High Power Lasers (PLD): (2017)

5. Jaworski, P., Koziol, P., Krzempek, K., Wu, D.K., Yu, F., Bojes, P., Dudzik, G., Liao, M.S., Abramski, K., Knight, J.: Antiresonant Hollow-Core Fiber-Based Dual Gas Sensor for Detection of Methane and Carbon Dioxide in the Near- and Mid-Infrared Regions. Sensors. 20(14), 3813 (2020). doi:10.3390/s20143813

6. Harrington, J.A.: Infrared fibers and their applications. SPIE Press (2004) 
7. Shi, Y.W., Abe, Y., Matsuura, Y., Miyagi, M.: Low loss smart hollow waveguides with new polymer coating material. Opt. Laser Techno. 31(2), 135-140 (1999). doi:10.1016/s0030-3992(99)00017-1

8. Harrington, J.A.: A review of IR transmitting, hollow waveguides. Fiber Integrated Opt. 19(3), 211-227 (2000). doi:10.1080/01468030050058794

9. Yu, T., Zuo, X., Liu, W.W., Gong, C.: $0.1 \mathrm{THz}$ super-resolution imaging based on 3D printed confocal waveguides. Opt. Commun. 459, 124896 (2020). doi:10.1016/j.optcom.2019.124896

10. Tichindelean, T.: Hollow glass fiber for $\mathrm{CO}_{2}$ laser surgery has coincident aiming beam. Laser Focus World 52(1), 117-119 (2016)

11. Van Den, B.H., Cornaz, P.F., Wagnieres, G.: Device for injecting the light energy of a laser beam into a fibre-optic optical waveguide and a method for adjusting and monitoring the position of the end of the fibre-optic optical waveguide. United States patent. US 5(117), 474 (1992)

12. Graham, C.H., Bartlett, N.R.: The relation of size of stimulus and intensity in the human eye: II. Intensity thresholds for red and violet light. J. Exp. Psychol. Gen. 24(6), 574-587 (1939)

13. Sheinis, A.I., Cozean, C., Forkner, J.F., Colles, M.J.: Optics for medical laser. United States patent. US 5, 289,557 (1994)

14. Rabii, C.D., Harrington, J.A.: Optical properties of dual-core hollow waveguides. Appl. Optics. 35(31), 6249-6252 (1996). doi:10.1364/ao.35.006249

15. Jing, C., Kendall, W., Harrington, J.A.: A simple way to establish a dual-core hollow fiber for laser surgery applications. Proceedings of the SPIE-Progress in Optical Fibers and Sensors for Medical Diagnostics and Treatment Applications 9702,97020D: (2016)

16. Picciolo, L.C., Murata, H., Kafafi, Z.H.: Organic light-emitting devices with saturated red emission using 6,13-diphenylpentacene. Appl. Phys. Lett. 78(16), 2378-2380 (2001). doi:10.1063/1.1362259

17. Allen, J.W.: Organic Electroluminescence and Competing Technologies. J. Lumin. 60-1, 912-915 (1994). doi:10.1016/0022-2313(94)90310-7

18. Navarro-Cía, M., Melzer, J.E., Harrington, J.A., Mitrofanov, O.: Silver-coated teflon tubes for waveguiding at 1-2 THz. J. Infrared Millim. Terahertz Waves. 36(6), 542-555 (2015). doi:10.1007/s10762-015-0157-5

19. Yang, Z.X., Zhong, W., Deng, Y., Au, C., Du, Y.-W.: Fabrication and Optical Behaviors of Core-Shell ZnS Nanostructures. Nanoscale Res. Lett. 5(7), 1124-1127 (2010). doi:10.1007/s11671-010-9612-3

20. Liang, G.J., Yi, M., Hu, H.B., Ding, K., Wang, L., Zeng, H.B., Tang, J., Liao, L., Nan, C.W., He, Y.B., Ye, C.H.: Coaxial-Structured Weavable and Wearable Electroluminescent Fibers. Adv. Electronic Mater. 3(12), 1700401 (2017). doi:10.1002/aelm.201700401

21. Rabii, C.D., Gibson, D.J., Harrington, J.A.: Processing and characterization of silver films used to fabricate hollow glass waveguides. Appl. Optics. 38(21), 4486-4493 (1999). doi:10.1364/ao.38.004486

22. Sun, Z., Fu, X., Li, G., Zhang, W., Zhong, Y., Wang, X., Jing, C., Lu, X., Yue, F., Chu, J.: Metallic hollow waveguide based on $\mathrm{GeO}_{2}-\mathrm{NaOH}$ precursor solution for transmission of $\mathrm{CO}_{2}$ laser radiations. Opt. 
Quant. Electron. 50(11) (2018). doi.: 10.1007/s11082-018-1662-2

23. Hou, L., Haji, M., Marsh, J.H., Bryce, A.C.: $10 \mathrm{GHz}$ AlGalnAs/InP $1.55 \mu \mathrm{m}$ passively mode-locked laser with low divergence angle and timing jitter. Opt. Express. 19(26), 75-80 (2011).

doi:10.1364/oe.19.000b75

24. Hidaka, T.: Loss calculations of the hollow-core, oxide-glass-cladding, middle-infrared optical waveguides. J. Appl. Phys. 53(1), 93-97 (1982)

25. Matsuura, Y., Abel, T., Hirsch, J., Harrington, J.A.: Small-bore hollow wave-guide for delivery of near singlemode IR laser-radiation. Electron. Lett. 30(20), 1688-1690 (1994). doi:10.1049/el:19941124

\section{Figures}

(a)

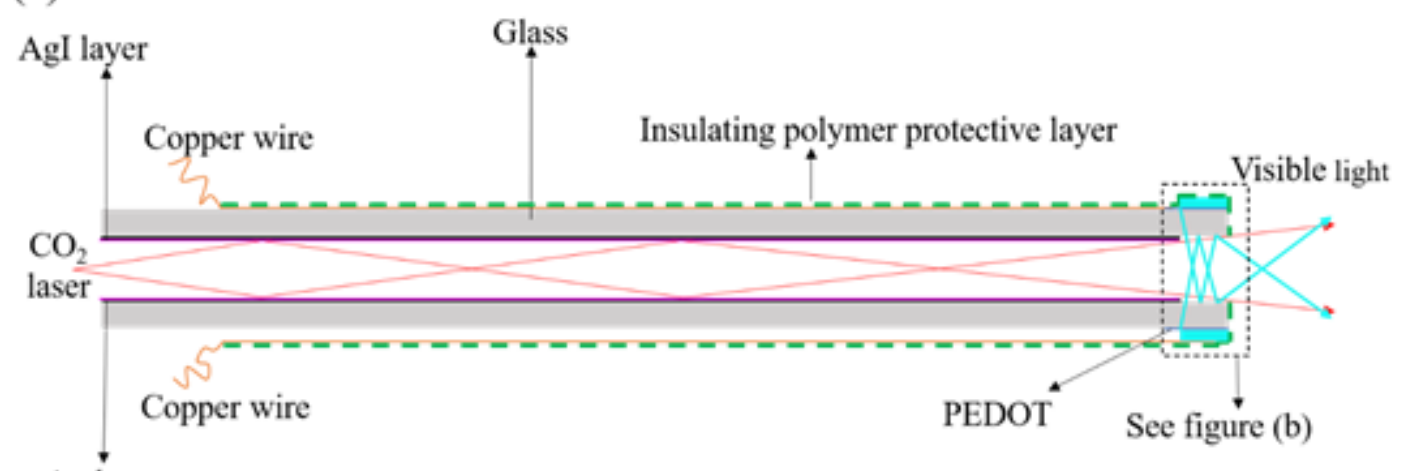

Ag layer

(b)

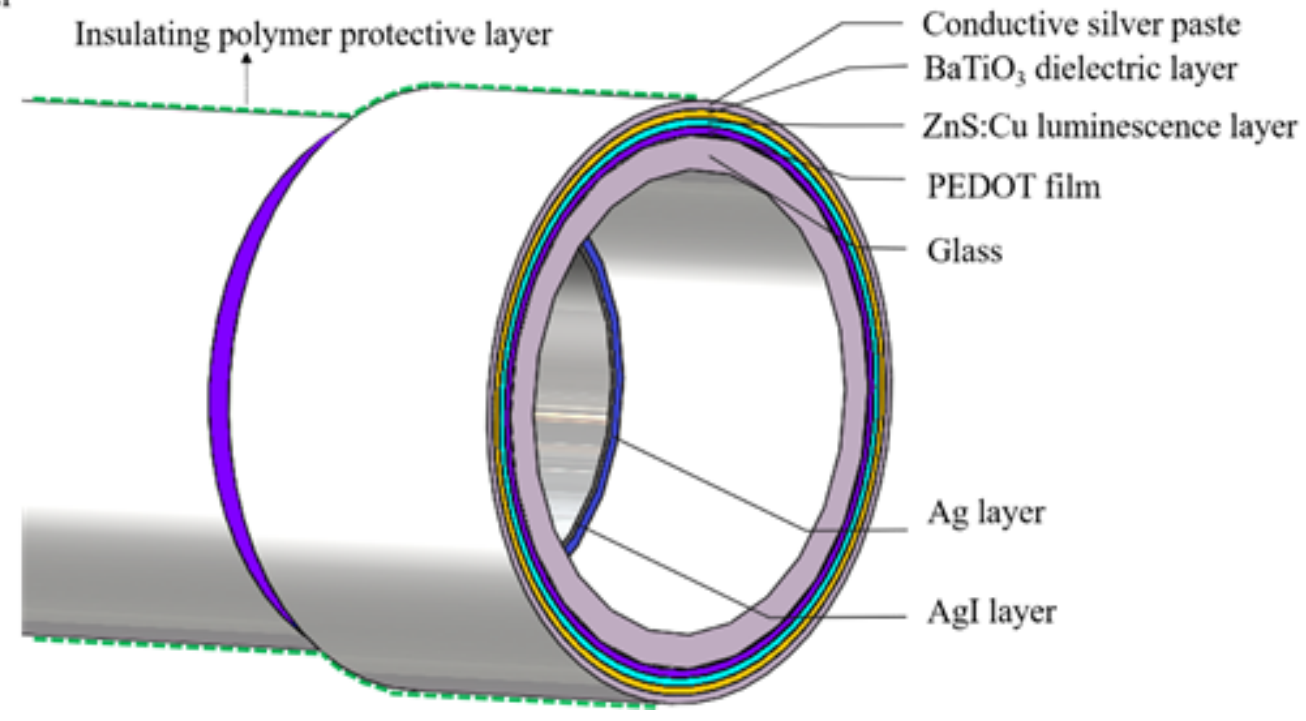

Figure 1

(a) EL structure-integrated mid-IR hollow optical fiber. (b) Output end structure of the fiber. 
(a)

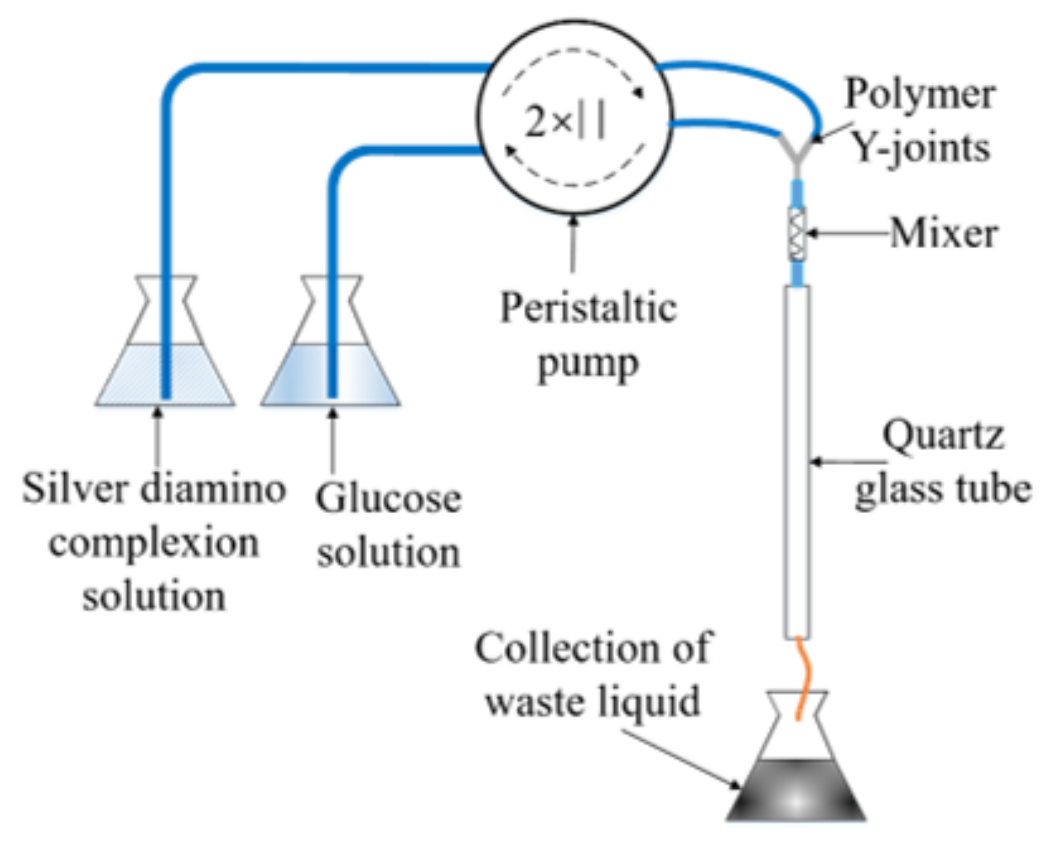

(b)

Upward pulling

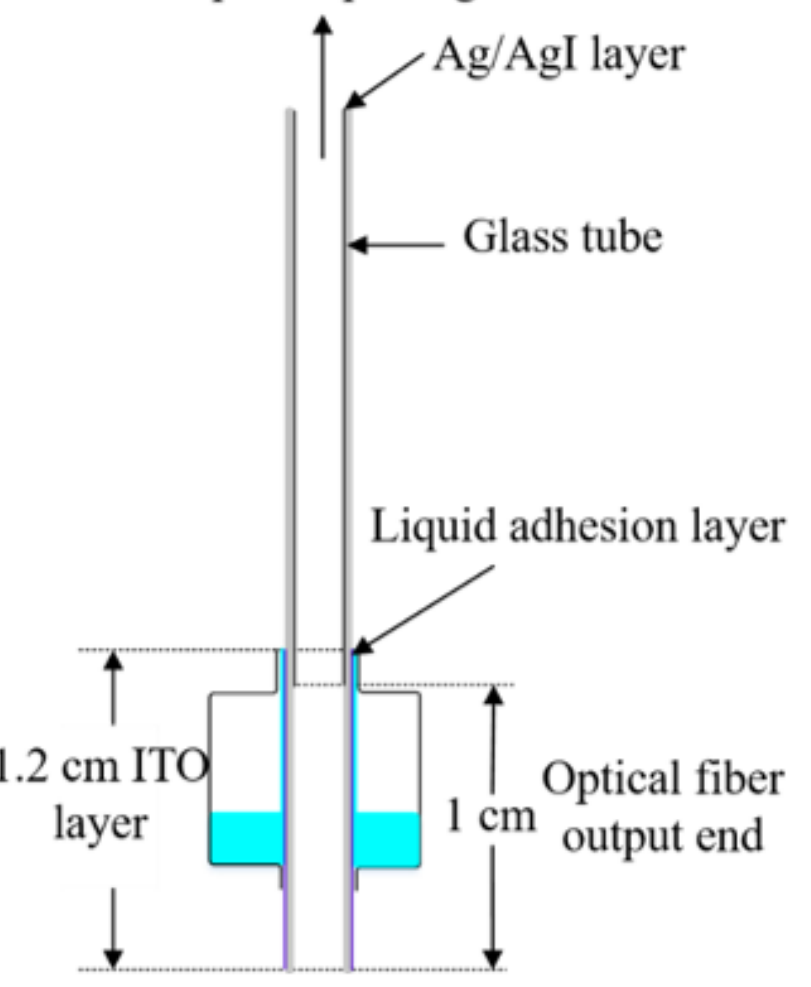

Figure 2

(a) Schematic DLPD method to prepare Ag/Agl hollow optical fiber device. (b) Dip-coating method.

Figure 3

(a) EDS element mapping of Zn, S, Cu for ZnS: Cu powder; (b) Cross-sectional SEM morphology of the EL structure at the end of the EL structure-integrated mid-IR hollow optical fiber. 


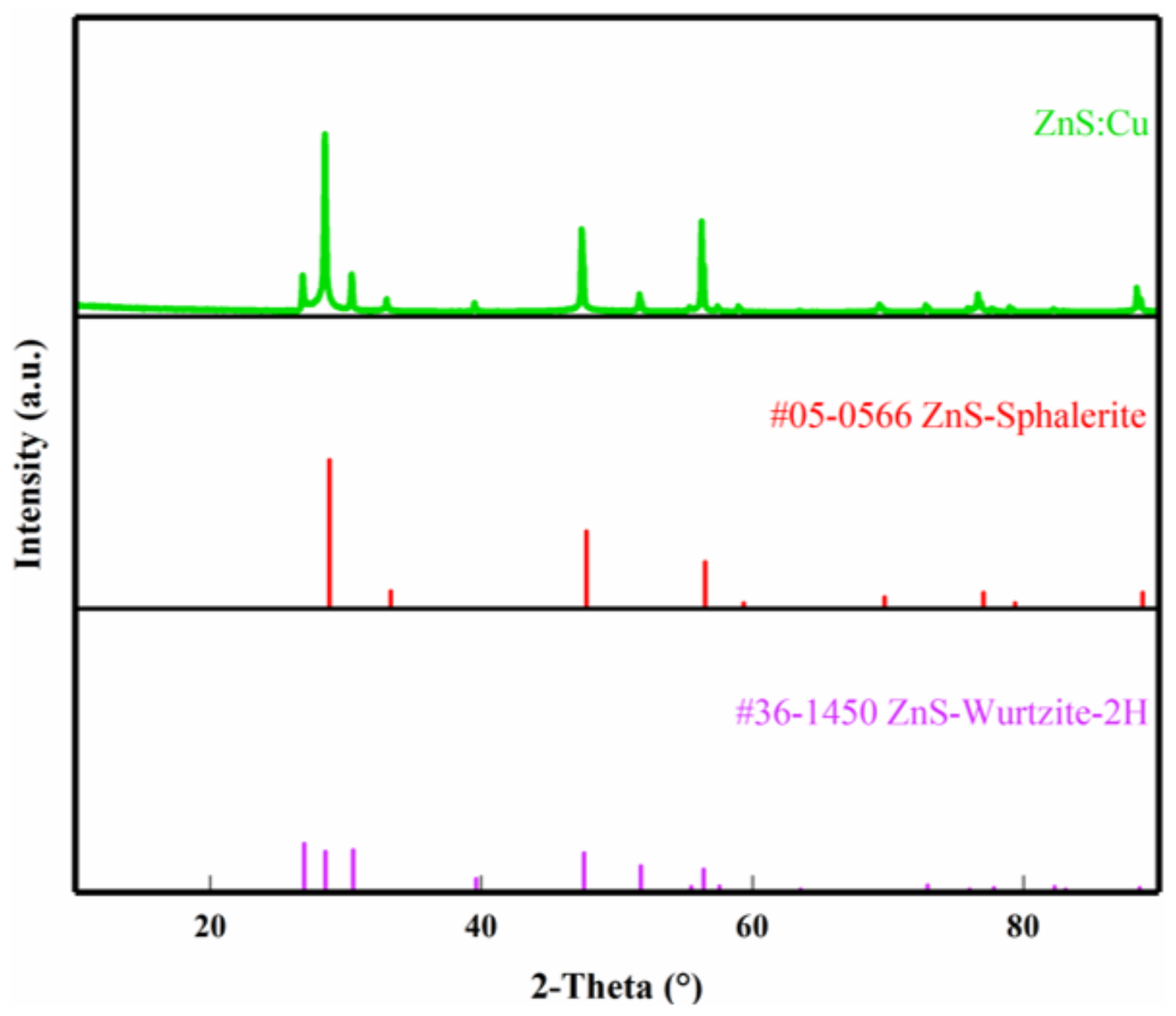

Figure 4

XRD pattern of ZnS: Cu phosphor. 
(a)

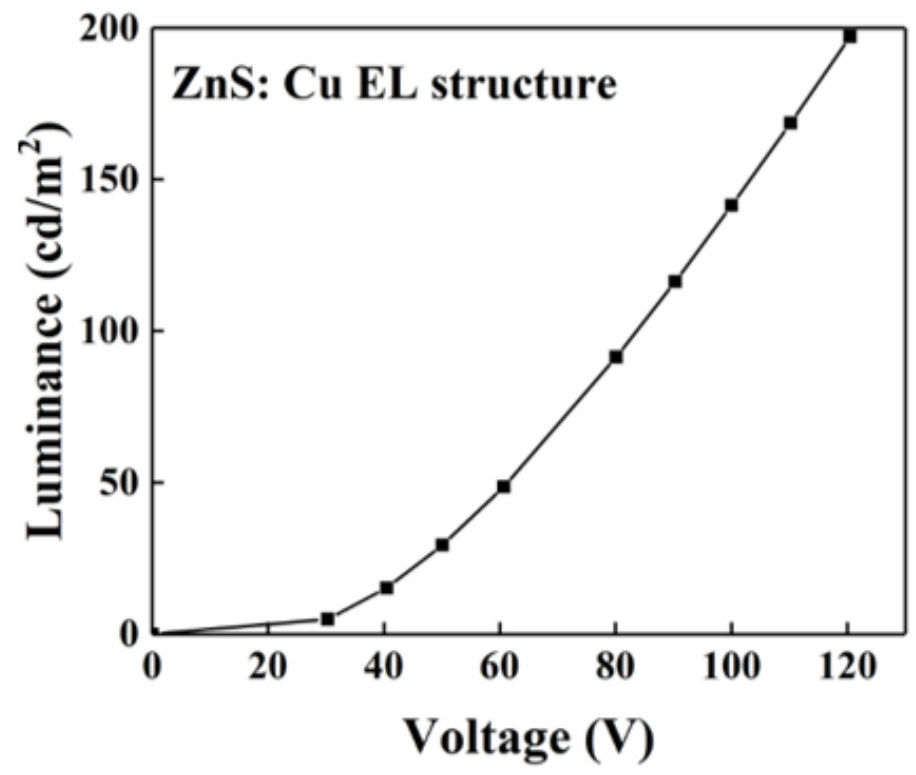

(b)

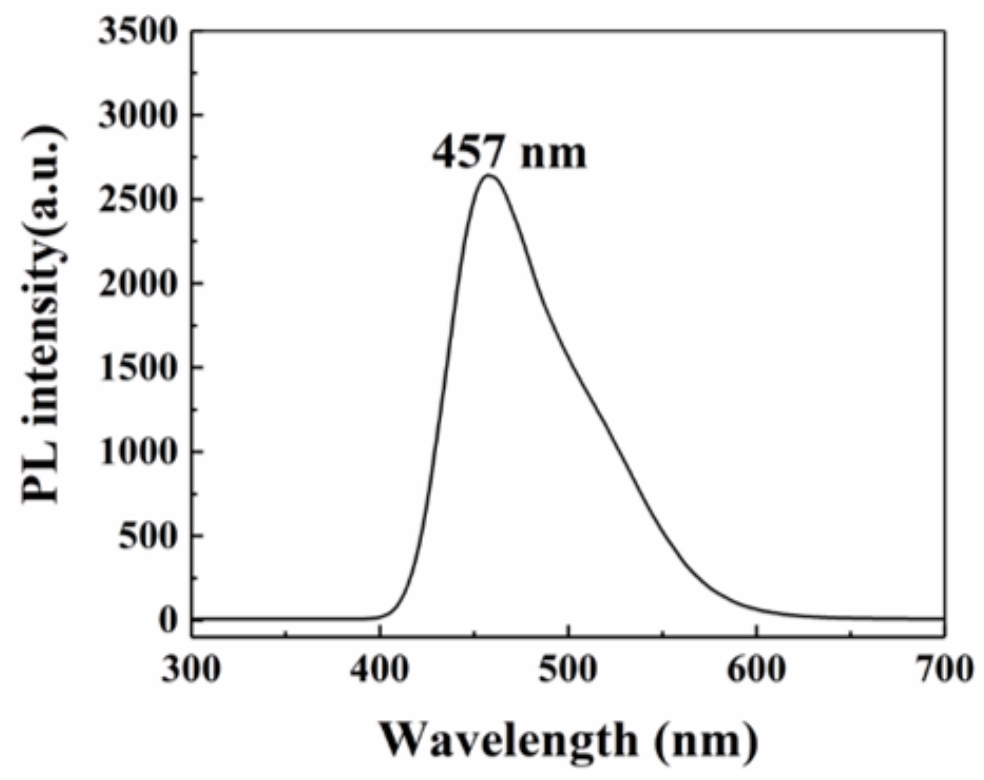

Figure 5

(a) Visible light EL luminance intensity of EL structure upon a different driving voltage. (b) EL spectrum of the EL structure-integrated mid-IR hollow optical fiber. 


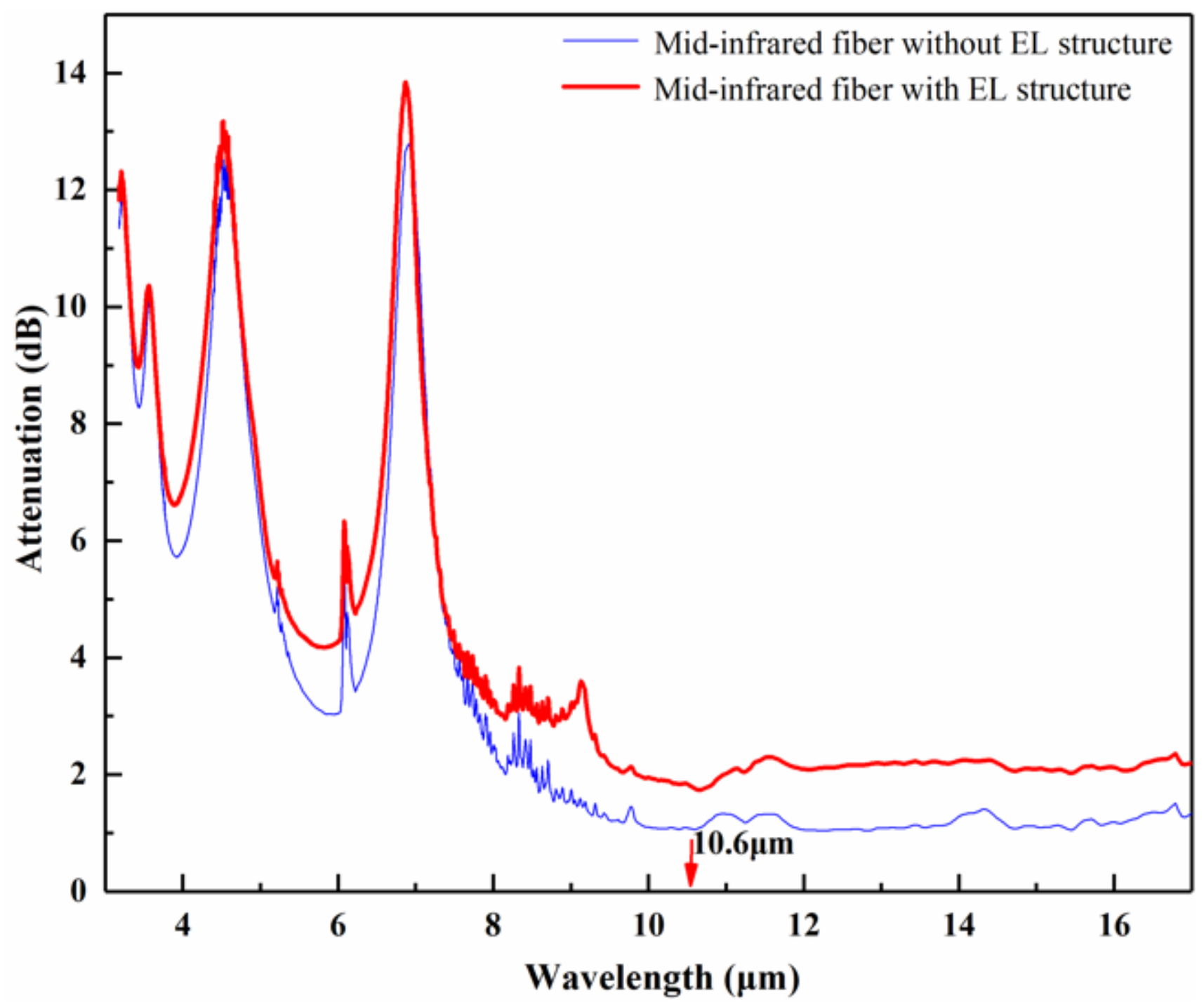

Figure 6

Loss spectra of the mid-IR fibers with and without the EL structure.

\section{Figure 7}

2D and 3D energy distribution profiles of the $\mathrm{CO}_{2}$ laser beam transmitted by the hollow optical fiber with an inner diameter of $530 \mu \mathrm{m}$. The output light energy distribution of the mid-IR fiber without the EL structure is in (a) and (b). The output light energy distribution of the mid-IR fiber with the EL structure is in (c) and (d). 


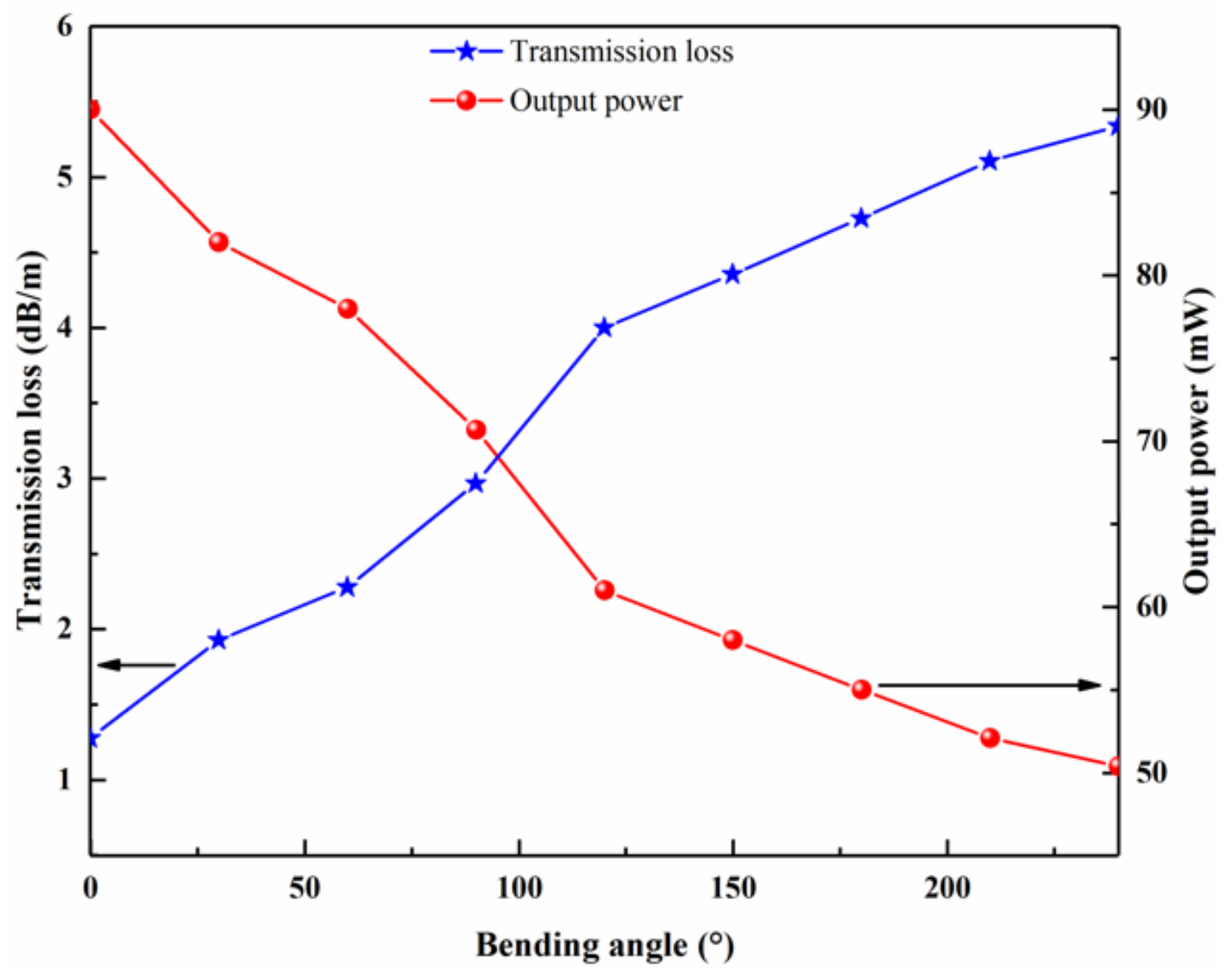

Figure 8

Transmission loss and output power of EL structure-integrated mid-IR hollow optical fiber with an internal diameter of $530 \mu \mathrm{m}$ at different bending angles (with the fixed curvature radius of $10 \mathrm{~cm}$ ) 


\section{Figure 9}

Simulation of surgery burning experiment of EL structure-integrated mid-IR hollow optical fiber. (a) Waveguide sample protected with a heat shrinkable tube. (b) Visible light spot for indication before $\mathrm{CO}_{2}$ laser emission. (c) Visible light spot and $\mathrm{CO}_{2}$ laser burning spot during $\mathrm{CO}_{2}$ laser emission. 